\title{
The Algorithm of Multi-sensor Data Fusion Based on the Modified Reciprocal Fuzzy Neartude
}

\author{
Qiang QU ${ }^{1, a}$, Xinhua HE ${ }^{1, b}$ and Weichao ZHANG $^{1, a}$ \\ ${ }^{1}$ Department of Information Engineering, Academy of Armored Force Engineering, Beijing 100072, \\ China \\ a535565543@qq.com, bhxh717495@sina.com
}

\begin{abstract}
Keywords: Sensor, Data fusion, Fuzzy neartude, Distance neartude, Fuzzy weighted fusion, Fuzzy membership.

Abstract. In order to improve the accuracy and reliability of the multi-sensor data fusion, a new modified reciprocal fuzzy neartude based approach to calculate the weights of the fusion model is proposed. Through the research of the fusion model, it is found that the fuzzy neartude is more practical than fuzzy membership for the calculation of the weights. The fusion performance of the five types of fuzzy neartude is analyzed, and the reciprocal fuzzy neartude is proved to be one of the best for the resolution and mount of calculation. However, the neartude cannot suppress the singular data well. To address the problem, the reciprocal fuzzy neartude is modified. The simulation analysis shows that the modified reciprocal fuzzy neartude based approach can fuse the multi-sensor data with high accuracy and reliability.
\end{abstract}

\section{Introduction}

According to the topology of the sensor data fusion modules, the sensor data fusion can be divided into distributed fusion and centralized fusion; according to the difference of input data types, it can be divided into homogeneous data fusion and heterogeneous data fusion; according to the different levels of the fusion module in sensor system, it can be divided into data level fusion, feature level fusion and decision level fusion [1].

The sensor data fusion algorithm includes the least squares algorithm, fuzzy weighted fusion algorithm , Calman filtering algorithm [2], clustering algorithm [3], neural network algorithm[4], template method[5] and D-S evidence reasoning algorithm[6] and so on, as shown in table 1. Fuzzy weighted fusion algorithm is to use fuzzy mathematics method for determining the weights of sensors in data fusion, and with strong applicability, moderate calculation cost and high precision, it is commonly used in the data level fusion, especially for homogeneous data. Some researchers[7] use fuzzy membership to solve the weights, but the membership degree solution needs a priori knowledge, which sometimes can not be obtained. To solve this problem, Li Xiong[8] adopted the fuzzy neartudeas as the fusion weight. In addition, Zhang Jun[9] adopted the correlation function in fuzzy theory to determine the weights and achieve satisfied fusion accuracy.

\section{Fuzzy weighted fusion method}

Fuzzy Processing of Sensor Data.In the ordinary set, we use the characteristic function U (x) to represent the set $U$. The $U(x)=1$ indicates that the ele/ment $x$ belongs to the set $U$, and the $U(x)=0$ indicates that the element does not belong to the set $U$. The characteristic function here can only take 0 and 1 , two values. If the value of the characteristic function extends from the set $\{0,1\}$ to the continuous value on the interval $[0,1]$, then we get the fuzzy set. The closer the eigenvalue of the element is to 1 , the greater the possibility to which the element belongs to the fuzzy set; and the smaller the opposite. The eigenfunction in fuzzy set theory is also called the membership function. 
The eigenvalue is called the membership degree, which represents the support degree of the element to the concept of the set.

Suppose that $\mathrm{N}$ sensors are used to measure an object, and the measurement data set is $\left\{\boldsymbol{A}_{1}, \boldsymbol{A}_{2}, \ldots, \boldsymbol{A}_{\mathrm{n}}\right\}$, where the measured data of a single sensor is a m-dimensioned vector $\boldsymbol{A}_{\mathrm{i}}=\left(x_{i 1}, x_{i 2}, \ldots\right.$, $\left.x_{i m}\right)$. Because of the error between the measured data and the real value, the two dimensional concept of "whether the measurement data is valid" is blurred, and the fuzzy sets of measurement data are defined. For $\boldsymbol{A}_{l}$ in the measurement data set, it is not possible to say whether it belongs to the fuzzy set, but to some extent the fuzzy set. This degree of membership reflects the validity of the sensor measurement data, that is, the proximity between the measured data and the real value.

Suppose that the membership degree of the elements in the above fuzzy sets is $a_{1}, a_{2}, \ldots, a_{n}$, where $a_{i}$ is the real number on the $[0,1]$ interval, then the Zadeh vector expression for the fuzzy sets of the measured data is [10] as follows:

$$
\AA^{\circ}=\left\{\frac{a_{1}}{A_{1}}, \frac{a_{2}}{A_{2}}, \ldots, \frac{a_{\mathrm{n}}}{\boldsymbol{A}_{n}}\right\}
$$

Fuzzy Weighted Fusion Model. The general average fusion model is to make the arithmetic average of the data from multi-sensor, that is

$$
\boldsymbol{Y}=\frac{\boldsymbol{A}_{1}+\boldsymbol{A}_{2}+\ldots+\boldsymbol{A}_{n}}{n}
$$

This fusion model does not take into account the differences in accuracy and reliability of each sensor measurement, but gives each sensor the same weight, i.e., $1 / \mathrm{n}$. In fact, we want to give the more accurate and reliable sensors the greater weight when weighted average is used. The fuzzy membership degree discussed above reflects the proximity between the measured data and the real value of the sensor, so it can be used as the weight.

$$
\boldsymbol{Y}=\frac{a_{1} \boldsymbol{A}_{1}+a_{2} \boldsymbol{A}_{2}+\ldots+a_{n} \boldsymbol{A}_{n}}{\sum_{i=1}^{n} a_{i}}
$$

The formula (2) shows that the membership degree is the key to the fuzzy weighted fusion method. Delphi method, fuzzy statistics method, increment method and factor weighting synthesis method are the main methods to solve the membership degree [10]. No matter what method is used to solve the degree of membership, either the subjective experience of experts or a large number of experimental data are needed, and most of the time, these conditions are not met. At the same time, the fuzzy-neartude-based fusion model can solve this problem.

Fusion Model Based on Fuzzy Neartude.In fuzzy set theory, fuzzy neartude refers to the proximity between two fuzzy sets, the axiomatic definition of it is: for fuzzy sets (or fuzzy vector) $\boldsymbol{A}, \boldsymbol{B}, \boldsymbol{C}$, assume there is a mapping $R$ which satisfy $F(U) \times F(U) \rightarrow[0,1]$ and follows:

(1) $R(\boldsymbol{A}, \boldsymbol{B})=R(\boldsymbol{B}, \boldsymbol{A})$

(2) $R(\boldsymbol{A}, \boldsymbol{A})=1$

(3) if $\boldsymbol{A} \supseteq \boldsymbol{B} \supseteq \boldsymbol{C}$ or $\boldsymbol{A} \subseteq \boldsymbol{B} \subseteq \boldsymbol{C}$, Then $R(\boldsymbol{A}, \boldsymbol{B}) \geqq R(\boldsymbol{A}, \boldsymbol{C})$

Then $R(A, B)$ is the fuzzy neartude of $A$ and $B$. When $A$ is a vector of sensor measurement data, and $\boldsymbol{B}$ for the vector of real value, $\mathrm{R}(\boldsymbol{A}, \boldsymbol{B})$ reflects the proximity between sensor measurement data and real value. In practical applications, the real value vector $\boldsymbol{B}$ is not known, and the average of multi-sensor measurements is used as its approximate estimate. Therefore, the weighted fusion model based on fuzzy neartude is defined as follows:

$$
\boldsymbol{Y}=\frac{r_{1} \boldsymbol{A}_{1}+r_{2} \boldsymbol{A}_{2}+\ldots+r_{n} \boldsymbol{A}_{n}}{\sum_{i=1}^{n} r_{i}}
$$

Among them, $r_{i}=R\left(\boldsymbol{A}_{i}, \boldsymbol{B}\right), \boldsymbol{A}_{i}=\left(x_{i 1}, x_{i 2}, \ldots, x_{i m}\right), \boldsymbol{B}=\left(\bar{x}_{1}, \bar{x}_{2}, \ldots, \bar{x}_{m}\right), \bar{x}_{j}=\sum_{i=1}^{n} x_{i j}$. 


\section{Fuzzy Neartude and Its Caculation}

Comparison of Commonly Used Fuzzy Neartude.The mapping that satisfies the definition of fuzzy neartude is not unique, so there are many formulas for it. Several commonly used fuzzy neartude is compared. Suppose $\boldsymbol{A}=\left(x_{1}, x_{2}, \ldots, x_{n}\right), \boldsymbol{B}=\left(y_{1}, y_{2}, \ldots, y_{n}\right)$, wherein $x_{i} \geq 0, y_{i} \geq 0$.

\section{Distance Neartude}

Distance neartude has three kinds, including reciprocal, logarithmic and subtraction type[11].

$$
\begin{aligned}
& R_{d l}\left(\begin{array}{ll}
A, B
\end{array}\right)=\frac{1}{1+\left|d_{A B}\right|} \\
& R_{d 2}(A, B)=\exp \left(-\left|d_{A B}\right|\right) \\
& R_{d 3}(A, \quad B)=1-\frac{\left|d_{A B}\right|}{\max \left(\left|d_{A B}\right|\right)}
\end{aligned}
$$

Where $d_{\boldsymbol{A} \boldsymbol{B}}$ refers to the distance between the fuzzy vector $\boldsymbol{A}$ and $\boldsymbol{B}$, which can be Hamming distance, Enclid distance, Hausdorf distance, Chebyshev distance, and so on. Which distance is chosen depends on the specific situation, where the Hamming distance is defined as

$$
d_{A B}=\sum_{i=1}^{n}\left|x_{i}-y_{i}\right|
$$

Hamming distance is simple in structure and low in computation. Although the resolution is not high, it is enough to meet most application requirements.

\section{Lattice Neartude [12]}

$$
R_{g}(A, B)=(A \otimes B) \wedge(A \text { e } B)^{C}
$$

$\boldsymbol{A} \otimes \boldsymbol{B} @ \stackrel{n}{V}_{i=1}^{V}\left(x_{i} \wedge y_{i}\right),(\boldsymbol{A} \text { e } \boldsymbol{B})^{C}=1-\boldsymbol{A}$ e $\boldsymbol{B}=\Lambda_{i=1}^{n}\left(x_{i} \vee y_{i}\right)$

In which $x_{i}$ and $y_{i} \in[0,1]$ 。 Lattice operation symbols " $\wedge$ " and " $\vee$ " that refers to the minimum and maximum operations respectively.

Maximum and Minimum Neartude [10]

$$
R_{m}(\boldsymbol{A}, \boldsymbol{B})=\frac{\sum_{i=1}^{n}\left(x_{i} \wedge y_{i}\right)}{\sum_{i=1}^{n}\left(x_{i} \vee y_{i}\right)}
$$

Arithmetic Mean Minimum Neartude [10]

$$
R_{s}\left(\begin{array}{l}
A, \boldsymbol{B}) \\
\sum_{i=1}^{n}\left(x_{i}+y_{i}\right)
\end{array}\right.
$$

\section{Geometric Mean Minimum Neartude[10]}

$$
R_{j}(\boldsymbol{A}, \boldsymbol{B})=\frac{\sum_{i=1}^{n}\left(x_{i} \wedge y_{i}\right)}{\sum_{i=1}^{n} \sqrt{x_{i} \cdot y_{i}}}
$$

In the above five types of fuzzy neartude, there is a serious defect in the Lattice Neartude: the condition of $R_{g}(\boldsymbol{A}, \boldsymbol{A})=1$ defined by the fuzzy neartude is not completely satisfied. In fact, the definition is only satisfied when $\boldsymbol{A}=0$ and $x_{i}=0$. To solve this problem, Jiang Hao[15] make some excellent work and provide a Modified Lattice Neartude as follows:

$$
R_{g}^{\prime}(\boldsymbol{A}, \boldsymbol{B})=\mid 1-\frac{1}{2}(\overline{\boldsymbol{A}}-\boldsymbol{A}+\overline{\boldsymbol{B}}-\boldsymbol{B})+\boldsymbol{A} \otimes \boldsymbol{B}-\boldsymbol{A} \text { e } \boldsymbol{B} \mid
$$


The ideal proximity should have the characteristics of high resolution, small calculation and being able to reject singular data. Because larger calculation and poor rejection of the singular data, the lattice neartude, the maximum and Minimum neartude, the arithmetic mean minimum neartude, and the geometric mean minimum neartude are not popular as to the distance neartude.

Modified Reciprocal Distance Neartude.The 3 kinds of distance neartude are compared in our research. For the amount of calculation, the subtractive distance neartude is maximum, the reciprocal distance neartude is minimum, and the exponential distance neartude is middle; for the resolution, the highest is the exponential one, then the reciprocal, and the subtractive the lowest; For the singular data reduction, the exponential one is the best then the subtractive, and the reciprocal is the worst. Therefore, without considering the amount of calculation, the exponential distance neartude degree is the best.

Although reciprocal distance neartude has the minimum amount of computation, but its singular data rejection ability is poor, and it needs a threshold to eliminate the singular data before fusion. The threshold value is normally selected by measuring environment and expert experience, so it is not practical and reliable. In order to solve this problem, the reciprocal distance neartude can be modified as follows:

Where $M$ is the distance amplification index, greater than 1 . In general, the larger the $M$ value, the stronger the singular value rejection capability and the higher the resolution. However, if the $M$ value is too large, it will affect the fusion accuracy. When the $\mathrm{M}$ takes the appropriate value, the modified reciprocal distance neartude can not only fuse data with more reliablity, but also with higher accuracy.

\section{Simulation Examples and Analysis}

Referring to the data in document [11], the fuzzy weighted average fusion method is simulated and analyzed.

Group 1: 852.0, 848.1, 851.9, 849.3, 849.9, 848.3, 851.2.

Group 2: 852.0, 848.1, 851.9, 849.3, 849.9, 848.3, 851.2, 100.

Group 3: 852.0, 848.1, 851.9, 849.3, 849.9, 848.3, 851.2, 100, 300.

Data is the measurement result of 7 temperature sensors to an 850 degree thermostat at the same time. And 1 or 2 singular data are added to the obtained data.

The Hamming distance is selected. The weighted fusion model based on the subtractive distance neartude the reciprocal distance neartude, the exponential distance neartude and the modified reciprocal distance neartude $(M=10)$ is compared, and the fusion results are shown in table 2 . The neartude value is shown in Figure 1-3 when there are no singular values, 1 singular values and 2 singular values.

Table1 Fusion Results of each fuzzy neartude

\begin{tabular}{l|c|c|c}
\hline \multicolumn{1}{c|}{ Types } & Group 1 & Group 2 & Group 3 \\
\hline $\begin{array}{l}\text { exponential distance } \\
\text { neartude }\end{array}$ & 850.0352 & 848.5747 & 848.5747 \\
\hline $\begin{array}{l}\text { subtractive distance } \\
\text { neartude }\end{array}$ & 850.0682 & 850.0959 & $\mathbf{8 1 8 . 0 0 2 4}$ \\
\hline $\begin{array}{l}\text { reciprocal distance } \\
\text { neartude }\end{array}$ & 850.0697 & $\mathbf{8 3 4 . 9 4 4 0}$ & $\mathbf{8 0 0 . 5 1 4 0}$ \\
\hline $\begin{array}{l}\text { modified reciprocal } \\
\text { distance neartude } \\
(\mathrm{M}=10)\end{array}$ & 850.0416 & 849.8577 & 849.9396 \\
\hline
\end{tabular}




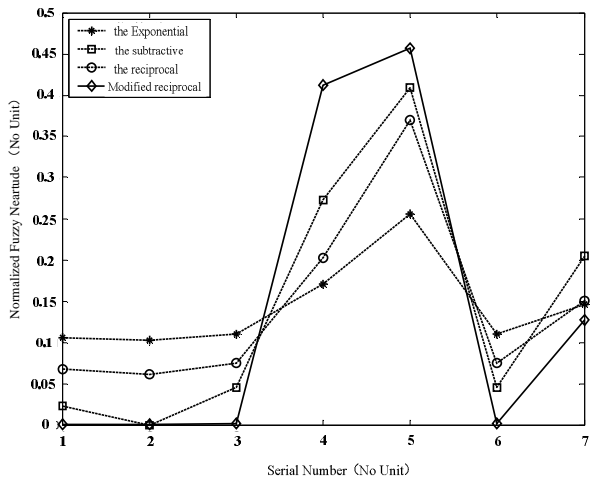

Fig 14 types of Fuzzy Neartude without singular data

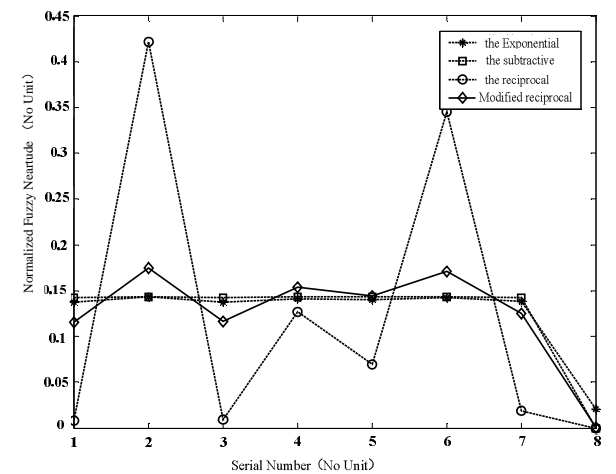

Fig 24 types of Fuzzy Neartude with 1 singular data

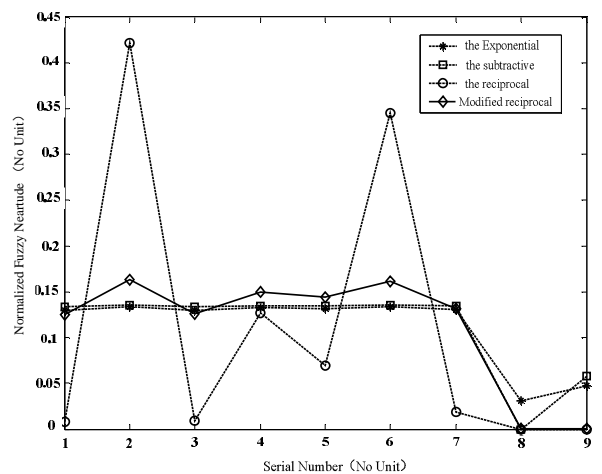

Fig 34 types of Fuzzy Neartude with 1 singular data

From simulation data above, we can see that when there is no singular data, all types of fuzzy neartude have good precision in fusion; when there is 1 singluar data, the fusion precision of all types were decreased, and the reciprocal decreased most; when there are 2 singular data, the precision of the subtractive and the exponential were decreased obviously, only the modified reciprocal is still high. The simulation analysis shows that the modified reciprocal fuzzy neartude has higher fusion accuracy and reliability.

\section{Conclusions}

Multi-sensor data fusion can make up for the shortage of single sensor measurement, improve measurement accuracy, reliability. Fuzzy weighted fusion method is a common method for similar data fusion in sensor data level.

In this paper, the theory of fuzzy weighted fusion is analyzed in detail. It is considered that the fuzzy neartude is used as the weight of the fusion model, which is more practical than the membership degree. Through the comparison of several commonly used fuzzy neartude, it is found that the reciprocal distance neartude has the highest resolution and the minimum amount of computation, but type of exponential and reciprocal type of distance close to the best performance, where the type of distance close to high resolution, minimum amount of computation, but for the bad suppression capability of singular data, its application is limited. In order to solve this problem, the modified reciprocal distance neartude is proposed by adding distance magnification index M. Simulation results show that the proposed method has obvious suppression effect on singular data, and has higher fusion accuracy and reliability. The research of this paper is not good enough. In the future, we will study (1) the influence of $M$ value on the fusion effect; (2) the problem of weight calculation in the multiplicative fusion model.

\section{Acknowledgements}

This work was financially supported by Academic Research Program of Academy of Armored Force Engineering. 


\section{References}

[1] Huang Man guo, Fan Shang chun, Zheng De zhi, et al. Multi Sensor Data Fusion Technology Research Progress. Journal of Sensor and Micro System. (2010) ,p. 5-8.

[2] Sun H, Wang W, Cao Y, et al: Application of Fuzzy Data Fusion in Multisensor Environment Monitor. (2009) , p. 550-553.

[3] Li Liang, Tan Shihai: Multi Sensor Data Fusion Method of. Clustering Based on Computer Engineering. (2013), p. 61-64.

[4] Zhou Guanming, Li Ronghui: Neural Network Based Sensor Network Data Fusion Technology. Journal of Computer Simulation.( 2011), p. 118-120.

[5] Prabhakar S, Jain A K. Decision-level fusion in fingerprint verification. (2002), p. 861-874.

[6] Yang Qiming, Zhang Ming: Pipeline Multi-sensor Data Fusion Based on D-S Evidence Theory. Journal of Oil and Gas Storage and Transportation. (2014), p. 252-254.

[7] Zhang Yanjun, Zhang Yanbin, Guo Deqing: The membership function of the data fusion method . Journal of Micro Computer Based Information. (2007), p.208-210.

[8] Zhang Jun, Yang Zichen: Data Fusion in Multisensor Data Acquisition Systems. sensors and microsystems. (2014), p.52-54.

[9] Li Angui, Zhang Zhihong, Meng Yan, et al: Fuzzy Mathematics and Its Applications: Metallurgical Industry Press. (2009).

[10] Fagui Liu, Zhao Juan: Fuzzy Closeness Degree and Its Applications. Journal of North China Institute of water conservancy and hydroelectric power. (2006), p.104-106.

[11] Wang Lingzhi: The Closeness Degree of Rough Fuzzy Sets. Journal of Sichuan Normal University (Natural Science Edition). (2002), p.476-478.

[12] Jiang Hao, Chen Sydney: Lattice Proximity Improvement, 2004. 